Chinese Journal of Organic Chemistry

ARTICLE

\title{
微波辐射下超细高岭土催化的肟类化合物的高效合成
}

\author{
梁玲牟佳玲陈悦王梦莎洪志* \\ (台州学院医药化工与材料工程学院 台州 318000)
}

\begin{abstract}
摘要 以廉价易得的超细高岭土为催化剂, 耐基类化合物和盐酸羟胺为原料, PEG-400 为溶剂, 在微波辐射的条件下高 效地合成一系列肟类化合物，同时探究了催化剂、溶剂种类、反应时间、反应温度等条件对反应的影响，并对所得的 产物进行了熔点、核磁、红外、质谱数据检测. 该方法具有反应时间短 $(5 \sim 15 \mathrm{~min})$ 、产率高、操作简便和环境友好等 优点. 另外，也初步探索了超细高岭土催化的微波辐射下肜交换反应及肜醚类化合物合成反应，产率分别为 $42 \%$ $67 \%$ 与 $59 \% \sim 72 \%$.
\end{abstract}

关键词 超细高岭土; 微波; 肜类化合物; 催化; 肜交换反应; 肜醚类化合物

\section{An Efficient Synthesis of Oxime Compounds Catalyzed by Superfine Kaolin under Microwave Irradiation}

\author{
Liang, Ling Mou, Jialing Chen, Yue Wang, Mengsha Hong, Zhi*
}

(School of Pharmaceutical and Materials Engineering, Taizhou University, Taizhou 318000)

\begin{abstract}
Oxime compounds were efficiently synthesized with aromatic or aliphatic ketones and hydroxylamine hydrochloride as raw materials in the PEG-400 solution, using cheap and readily available superfine kaolin as catalyst under microwave irradiation condition. The effects of the catalyst, the solvent type, reaction time, reaction temperature on the reaction were investigated. The structures of the products were detected by melting point, nuclear magnetic resonance, infrared spectroscopy and mass spectrometry data. This method has the advantages of short reaction times $(5 \sim 15 \mathrm{~min})$, good yields, and simple operation as well as environmental friendliness. In addition, the preliminary experiment shows that superfine kaolin could also catalyze transoximation reaction and preparation of oxime ether compounds under microwave irradiation with the yields of $42 \% \sim 67 \%$ and $59 \% \sim 72 \%$.

Keywords superfine kaolin; microwave; oxime compound; catalysis; transoximation; oxime ether compounds
\end{abstract}

肜是一类具有通式 $\mathrm{R}^{1} \mathrm{R}^{2} \mathrm{C}=\mathrm{NOH}$ 的有机化合物, 作为羰基的一种保护形式, 能在许多条件下保持稳 定 $^{[1,2]}$, 而且含有肜官能团的化合物都有一定的细胞毒 性, 大多具有除草 ${ }^{[3]}$ 、杀虫 ${ }^{[4]}$ 、抗病毒 ${ }^{[5]}$ 、抗肿瘤 $\left[{ }^{[6}\right.$ 等重 要的生物活性. 肜类化合物的合成方法较多 ${ }^{[7 \sim 10]}$, 但目 前所知的大多数肜类化合物都是由醛或酮与羟胺缩合 得 到 ${ }^{[11]}$, 此类 反应可以在 $\mathrm{AcONa}^{[12]}, \mathrm{NaOH}^{[13]}$, $\mathrm{C}_{5} \mathrm{H}_{5} \mathrm{~N}^{[14]}, \mathrm{NaHCO}_{3}{ }^{[15]}$ 和 $\mathrm{CH}_{3} \mathrm{ONa}^{[16]}$ 等碱性化合物作用 下进行, 也可以在路易斯酸 $\mathrm{FeCl}_{3} \cdot 6 \mathrm{H}_{2} \mathrm{O}^{[17]}, \mathrm{CuSO}_{4}{ }^{[18]}$, $\mathrm{CoCl}_{2}{ }^{[19]}$ 或质子酸 $\mathrm{H}_{2} \mathrm{SO}_{4}{ }^{[20]}, \mathrm{TsOH}^{[21]},(\mathrm{COOH})_{2}{ }^{[22]}$ 等催化 下发生, 但是大多存在反应时间长, 催化剂不易回收及 难以重复利用等缺点. 因此, 开发该类化合物的绿色合
成方法具有重要意义. 另外, 我们注意到 Juskowiak ${ }^{[23]}$, Shimaoka $^{[24]}$, Sridhar ${ }^{[25]}$ 与 Hyodo ${ }^{[26]}$ 等曾报道了一种以羰 基类化合物与酮肜或乙酰氧肟酸等为原料, 在质子酸或 路易斯酸催化下进行的肪交换反应，该类反应可以达到 较高的产率和 $Z / E$ 比值, 但较少被人们所关注.

高岭土是一种储量丰富的白色黏土类矿物, 是由氧 化铝和氧化硅结合成的层状结构的水合硅酸铝盐, 两者 比率影响其水悬浮液的 $\mathrm{pH}$ 值，一般在 $6.0 \sim 8.0$ 之间，既 有 Lewis 酸性也有 Brønst 酸性, 其复合化学分子式是 $\mathrm{Al}_{2} \mathrm{O}_{3} \cdot 2 \mathrm{SiO}_{2} \cdot 2 \mathrm{H}_{2} \mathrm{O}^{[27,28]}$. 由于高岭土的表面特性, 会发 生离子吸附作用, 可以方便地进行物理改性或化学改 性，也可作为多种杂多酸的载体，应用领域非常广

* Corresponding author. E-mail: zhihong@tzc.edu.cn

Received November 17, 2018; revised February 13, 2019; published online March 29, 2019.

Project supported by the Basic Public Research Project of Zhejiang Province (No. LGG18B020002).

浙江省基础公益研究计划(No. LGG18B020002)资助项目. 
泛 $^{[29,30]}$. 其中超细高岭土, 由于价廉易得、比表面积大 和吸附性强, 受到广泛关注 ${ }^{[31 ~ 33]}$, 但是较少直接将超细 高岭土作为催化剂应用于有机合成领域, 仅有的例子包 括葡萄糖异构化生成果糖 ${ }^{[27]}$, 芳基腈与叠氮化钠的环 合反应 ${ }^{[34]}$ 、甲醛水热条件下合成 $2,4,8,10$-四氧杂螺 [5.5] 十一烷 ${ }^{[35]}$ 、芳烃的氯化和溴化反应 ${ }^{[36]}$ 、酚类化合物的 $O$-甲基化反应 ${ }^{[37]}$ 和酚类化合物经 Ene 反应合成 $\gamma$-内酯 [38]等.

相对于一般的加热的方式, 微波辐射可以大幅提升 反应速度, 并具有操作简便、产率高、选择性好等优点, 目前在有机合成领域中的应用已经非常普遍 ${ }^{[39,40]}$. 最近 Lee $^{[41]}$, Erdogan $^{[42]}$, Mushtaque ${ }^{[43]}$ 等课题组分别报道了碱 性氧化铝、吡啶、醋酸钠催化下, 酮类化合物与盐酸着 胺在微波辐射下合成肟类化合物的反应. Chermahini 等 ${ }^{[34]}$ 在进行芳基腈与叠氮化钠的环合制备 5-取代基$1 H$-四氮唑时, 曾推断高岭土中的硅酸根 $\left[\mathrm{SiO}_{4}\right]^{4-}$ 能通过 活化氧基, 促进 1,3-偶极环加成反应. 类似的, 我们预 测高岭土对于羰基也有一定活化作用，能够促进羰基类
化合物与羟胺的缩合反应. 因此，我们尝试直接将价廉 易得的超细高岭土作为催化剂，在微波辐射下，在 PEG-400 溶液中, 进行醛或酮与盐酸差弪胺、烷氧基胺盐 酸盐的缩合反应以及酮类化合物与乙酰氧肟酸的肜交 换反应，简便高效地制备一系列肜类化合物及其肟醚衍 生物.

\section{1 结果与讨论}

\section{1 缩合反应条件的篮选}

我们取二苯甲酮 $(1 \mathrm{~s}, 5 \mathrm{mmol})$ 和盐酸着胺 $(10 \mathrm{mmol})$, 在微波辐射下合成二苯甲酮肜 $(2 \mathrm{~s})$, 考察溶剂种类、反应 时间、催化剂的种类及用量和微波功率对反应的影响, 结果列于表 1 中.

从表 1 可以看出, 当不加溶剂与催化剂, 微波功率 为 $130 \mathrm{~W}$ 时, 反应几乎不能够进行(表 1 , Entry 1); 当加 入 0.05 equiv. 的催化剂超细高岭土(Superfine kaolin, 平 均粒径 $5 \mu \mathrm{m}$ ) 时, 反应能进行, 但产率非常低(表 1 , Entry

表 1 合成肜类化合物 $2 \mathrm{~s}$ 反应条件的优化

Table 1 Optimization of reaction conditions for the synthesis of oxime compound $2 \mathrm{~s}$

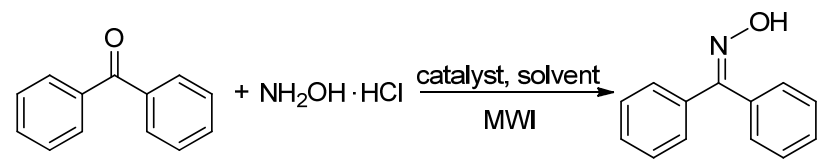

1s

$2 s$

\begin{tabular}{|c|c|c|c|c|c|}
\hline Entry & Solvent & Catalyst (molar equiv.) & Microwave power/W & Time/min & Isolated yield ${ }^{a} / \%$ \\
\hline 1 & - & - & 130 & 3 & Trace \\
\hline 2 & - & Superfine kaolin $(5 \mu \mathrm{m}, 0.05)^{b}$ & 130 & 3 & 10 \\
\hline 3 & Toluene & Superfine kaolin $(5 \mu \mathrm{m}, 0.05)$ & 130 & 3 & 24 \\
\hline 4 & Xylenes & Superfine kaolin $(5 \mu \mathrm{m}, 0.05)$ & 130 & 3 & 33 \\
\hline 5 & PEG-200 & Superfine kaolin $(5 \mu \mathrm{m}, 0.05)$ & 130 & 3 & 36 \\
\hline 6 & PEG-400 & Superfine kaolin $(5 \mu \mathrm{m}, 0.05)$ & 130 & 3 & 40 \\
\hline 7 & PEG-400 & Superfine kaolin $(5 \mu \mathrm{m}, 0.10)$ & 130 & 3 & 51 \\
\hline 8 & PEG-400 & Superfine kaolin $(5 \mu \mathrm{m}, 0.15)$ & 130 & 3 & 63 \\
\hline 9 & PEG-400 & Superfine kaolin $(5 \mu \mathrm{m}, 0.20)$ & 130 & 3 & 65 \\
\hline 10 & PEG-400 & Superfine kaolin $(5 \mu \mathrm{m}, 0.15)$ & 195 & 3 & 75 \\
\hline 11 & PEG-400 & Superfine kaolin $(5 \mu \mathrm{m}, 0.15)$ & 260 & 3 & 76 \\
\hline 12 & PEG-400 & Superfine kaolin $(5 \mu \mathrm{m}, 0.15)$ & 195 & 5 & 92 \\
\hline 13 & PEG-400 & Superfine kaolin $(5 \mu \mathrm{m}, 0.15)$ & 195 & 7 & 88 \\
\hline 14 & PEG-400 & Superfine kaolin $(11 \mu \mathrm{m}, 0.15)^{b}$ & 195 & 5 & 60 \\
\hline 15 & PEG-400 & Kaolin $(0.15)$ & 195 & 5 & 46 \\
\hline 16 & PEG-400 & - & 195 & 5 & 13 \\
\hline 17 & PEG-400 & $\alpha$-Alumina trihydrate $(0.15)$ & 195 & 5 & 20 \\
\hline 18 & PEG-400 & $\mathrm{Al}_{2} \mathrm{O}_{3}(0.15)$ & 195 & 5 & 15 \\
\hline 19 & PEG-400 & Nano- $\gamma-\mathrm{Al}_{2} \mathrm{O}_{3}(20 \mathrm{~nm}, 0.15)^{b}$ & 195 & 10 & Trace \\
\hline 20 & PEG-400 & $\mathrm{SiO}_{2}(200 \sim 300 \text { mesh, } 0.15)^{b}$ & 195 & 10 & Trace \\
\hline 21 & PEG-400 & $\mathrm{ZnO}(0.15)$ & 195 & 10 & 15 \\
\hline $22^{\mathrm{c}}$ & EtOH & Pyridine $(5 \mathrm{~mL})$ & 560 & 5 & 73 \\
\hline
\end{tabular}

${ }^{a}$. Reaction conditions: benzophenone (1s, $\left.5 \mathrm{mmol}\right)$, hydroxylamine hydrochloride $(10 \mathrm{mmol})$, PEG-400 $(10 \mathrm{~mL}){ }^{b} 5,11 \mu \mathrm{m}, 20 \mathrm{~nm}$ and $200 \sim 300 \mathrm{mesh}$ are the particle sizes of catalysts. ${ }^{c}$ Reaction conditions in literature [44]: benzophenone (1s, $\left.5.5 \mathrm{mmol}\right)$, hydroxylamine hydrochloride (14.4 mmol), solvent EtOH (5 mL), catalyst pyridine $(5 \mathrm{~mL})$. 
2). 考虑到微波反应对极性有机物进行选择性加热, 只 有具有较大分子偶极矩的有机分子才能被微波有效加 热, 由于极性分子内电荷分布不均匀, 故其介电常数较 大, 能与微波产生较强的偶合作用, 故该反应需要在极 性溶剂中进行. 从甲苯、二甲苯、聚乙二醇 200 (PEG200)和聚乙二醇 400 (PEG-400) 来看, 当溶剂为 PEG-400 时, 反应的产率达到 40\%(表 1, Entry 6). 当增加超细高 岭土用量、改变微波辐射的功率或延长反应时间, 结果 发现当超细高岭土用量为 0.15 equiv. 微波辐射的功率 为 $195 \mathrm{~W}$ 、反应时间为 $5 \mathrm{~min}$ 时, 产率达到 $92 \%$ (表 1 , Entry 12). 另外, 对比不加催化剂和其他固体催化剂如 粒径为 $11 \mu \mathrm{m}$ 的超细高岭土、化学纯高岭土、无催化剂、 酸性氧化铝 $\left(\alpha\right.$-Alumina trihydrate)、中性氧化铝 $\left(\mathrm{Al}_{2} \mathrm{O}_{3}\right)$ 、 纳米 $\gamma$-型氧化铝 (nano- $\gamma$ - $\mathrm{Al}_{2} \mathrm{O}_{3}$ ) (平均粒径 $20 \mathrm{~nm}$ )、 $\mathrm{SiO}_{2}$ (200 300 目)和 $\mathrm{ZnO}$ 的反应, 结果发现产率都不理想 (表 1, Entries 14 20). 另外, 参考文献[44]中报道的方 法, 产率为 $73 \%$ (表 1, Entry 22), 略低于本实验的优化 条件.

\section{2 反应底物拓展}

为了扩大反应的适应范围, 在上述优化反应条件的 基础上，合成了一系列肜类化合物，结果如表 2 所示.

由表 2 可以看出, 微波辐射条件下, 脂肪族的链状 酮或环状酮与盐酸着胺的反应产率较低(表 2 , Entries $1 \sim 6)$, 而带有苯环的醛或酮与盐酸着胺的反应状况良 好, 产率基本在 $80 \%$ 以上. 苯环上的电子效应对反应没 有明显的影响, 无论是吸电子取代(例如氟、氯或硝基) 还是供电子取代基(例如甲基、羟基、甲氧基)都能得到 满意的产率. 另外, 亚芳基丙酮肜(2r)分离得到较稳定 的 $Z 、 E$ 型异构体, 其中 $E$ 型的比例略高(表 2, Entry 18). 同时含杂环的芳香酮与盐酸羟胺的反应也能得到较满 意产率(表 2, Entries 11, 17).

\section{3 醛、酮活性比较}

为了探究醛、酮与盐酸着胺反应的相对选择性, 我 们投入苯甲醛 $(1 \mathrm{~g}, 5 \mathrm{mmol}) 、$ 苯乙酮 $(1 \mathrm{l}, 5 \mathrm{mmol})$ 与盐酸着 胺(10 mmol)反应(Eq. 1), 其它都按优化条件进行, 反应 进行 $5 \mathrm{~min}$ 后, 苯甲醛反应了 $78 \%$, 而苯乙酮几乎没

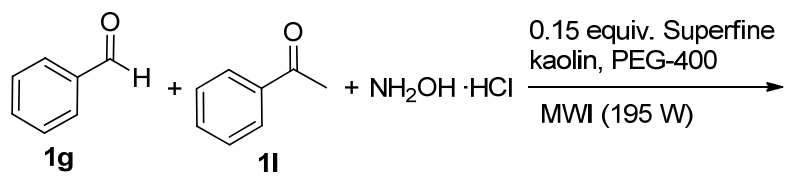<smiles>O/N=C/c1ccccc1</smiles><smiles>C/C(=N\O)c1ccccc1</smiles>

$78 \%$ yield
表 2 肟类化合物 $\mathbf{2 a} \sim 2 \mathrm{v}$ 的合成

Table 2 Synthesis of oxime compounds $\mathbf{2 a} \sim \mathbf{2 v}$

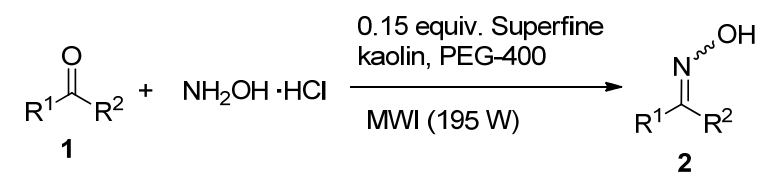

\begin{tabular}{|c|c|c|c|c|}
\hline Entry & $\mathrm{R}^{1}$ & $\mathrm{R}^{2}$ & Product & Isolated yield $/ \%$ \\
\hline 1 & $\mathrm{CH}_{3}$ & $\mathrm{CH}_{3}$ & $2 \mathbf{a}$ & 71 \\
\hline 2 & $\mathrm{CH}_{3}$ & $\mathrm{CH}_{3} \mathrm{CH}_{2}$ & $2 \mathbf{b}$ & 62 \\
\hline 3 & \multicolumn{2}{|c|}{ Cyclopentanone } & $2 c$ & 43 \\
\hline 4 & \multicolumn{2}{|c|}{ Cyclohexanone } & 2d & 48 \\
\hline 5 & \multicolumn{2}{|c|}{ 2-Methylcyclohexanone } & $2 \mathrm{e}$ & 52 \\
\hline 6 & \multicolumn{2}{|c|}{ Cycloheptanone } & $2 \mathrm{f}$ & 63 \\
\hline 7 & $\mathrm{H}$ & $\mathrm{C}_{6} \mathrm{H}_{5}$ & $2 \mathrm{~g}$ & 81 \\
\hline 8 & $\mathrm{H}$ & $2-\mathrm{HOC}_{6} \mathrm{H}_{4}$ & $2 \mathrm{~h}$ & 86 \\
\hline 9 & $\mathrm{H}$ & $4-\mathrm{HOC}_{6} \mathrm{H}_{4}$ & $2 \mathbf{i}$ & 42 \\
\hline 10 & $\mathrm{H}$ & $2,4-\mathrm{Cl}_{2} \mathrm{C}_{6} \mathrm{H}_{3}$ & $2 \mathbf{j}$ & 87 \\
\hline 11 & $\mathrm{CH}_{3}$ & 2-Furyl & $2 \mathbf{k}$ & 42 \\
\hline 12 & $\mathrm{CH}_{3}$ & $\mathrm{C}_{6} \mathrm{H}_{5}$ & 21 & 83 \\
\hline 13 & $\mathrm{CH}_{3}$ & $4-\mathrm{HOC}_{6} \mathrm{H}_{4}$ & $2 \mathrm{~m}$ & 87 \\
\hline 14 & $\mathrm{CH}_{3}$ & $4-\mathrm{CH}_{3} \mathrm{OC}_{6} \mathrm{H}_{4}$ & $2 n$ & 84 \\
\hline 15 & $\mathrm{CH}_{3}$ & 4- $\mathrm{NO}_{2} \mathrm{C}_{6} \mathrm{H}_{4}$ & 20 & 87 \\
\hline 16 & $\mathrm{CH}_{3}$ & 4- $\mathrm{ClC}_{6} \mathrm{H}_{4}$ & $2 p$ & 83 \\
\hline 17 & $\mathrm{CH}_{3}$ & 3-Pyridyl & $2 q$ & 77 \\
\hline 18 & $\mathrm{CH}_{3}$ & $\mathrm{C}_{6} \mathrm{H}_{5} \mathrm{CH}=\mathrm{CH}$ & $2 r$ & $48^{b} / 40^{c}$ \\
\hline 19 & $\mathrm{C}_{6} \mathrm{H}_{5}$ & $\mathrm{C}_{6} \mathrm{H}_{5}$ & $2 s$ & 92 \\
\hline 20 & $\mathrm{C}_{6} \mathrm{H}_{5}$ & $2-\mathrm{ClC}_{6} \mathrm{H}_{4}$ & $2 t$ & 89 \\
\hline 21 & $4-\mathrm{CH}_{3} \mathrm{OC}_{6} \mathrm{H}_{4}$ & $4-\mathrm{CH}_{3} \mathrm{OC}_{6} \mathrm{H}_{4}$ & $2 u$ & 86 \\
\hline 22 & $4-\mathrm{FC}_{6} \mathrm{H}_{4}$ & $4^{\prime}-\mathrm{FC}_{6} \mathrm{H}_{4}$ & $2 v$ & 91 \\
\hline
\end{tabular}

${ }^{a}$ Reaction conditions: ketones 1 (5 mmol), hydroxylamine hydrochloride (10 $\mathrm{mmol})$, superfine kaolin $(5 \mu \mathrm{m}, 0.75 \mathrm{mmol})$, PEG-400 $(10 \mathrm{~mL}), 195 \mathrm{~W}^{b}(Z, E)$ 3-Buten-2-one-4-phenyl oxime. ${ }^{c}(E, E)$-3-Buten-2-one-4-phenyl oxime.

有反应，也没有查尔酮生成. 实验表明酮类与醛类进行 竞争性肟化的时候，酮类的反应活性比醛类要弱; 另外， 在该反应条件下，反应底物并未经 Aldol 反应生成查尔 酮, 表明超细高岭土催化剂也具有较好的化学选择性.

另外, 以 1,3 -二乙酰基苯 $(3,5 \mathrm{mmol})$ 与盐酸着胺 $(15$ $\mathrm{mmol}$ )为底物(两者投料物质的量之比为 $1: 3$ ), 其它都 按优化条件进行，反应 $5 \mathrm{~min}$, 发现在该条件下产物以 1,1'-(1,3-亚苯基)二乙氧基二肜(4a) 为主，单取代的肜 (4b) 几乎没有(Eq. 2).

\section{4 肟交换反应制备肟类化合物}

在微波辐射下超细高岭土催化羰基类化合物与盐 酸着胺制备肜类化合物的实验取得一定结果的基础上, 


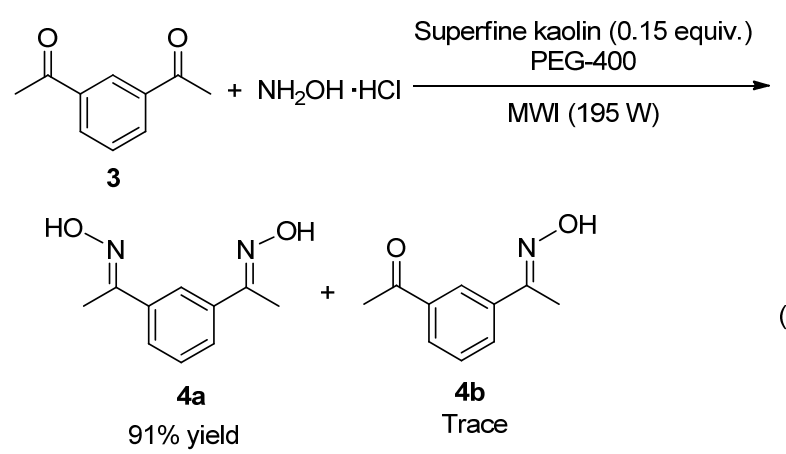

以二苯甲酮 $(1 \mathrm{~s})$ 为原料与环己酮䏡、丙酮肟、环戊酮肜 或乙酰氧肟酸 (5)反应, 制备二苯甲酮肜(2s), 对肜交换 反应做了初步的研究. 结果发现, 二苯甲酮与乙酰氧肜 酸(5)反应时, 产率最高(表 3).

表 3 肜交换反应底物 $\mathbf{2}$ 或 $\mathbf{5}$ 的篮选

Table 3 Screening of substrates $\mathbf{2}$ or $\mathbf{5}$ of the transoximation

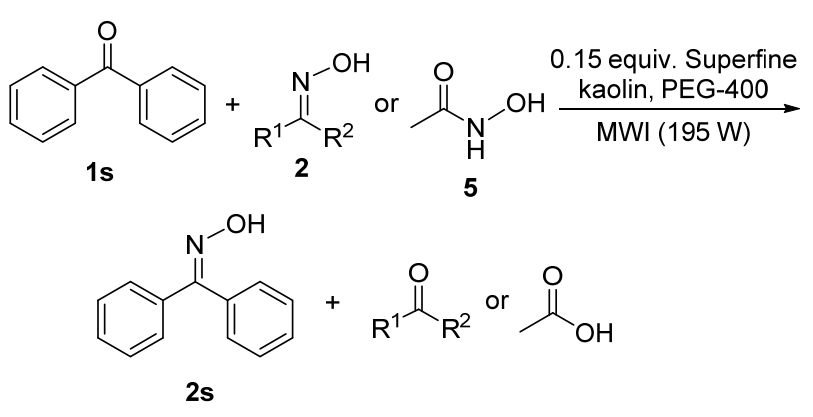

\begin{tabular}{lcc}
\hline Entry & Substrate $\mathbf{2}$ or $\mathbf{5}$ & Isolated yield/\% \\
\hline & \\
\end{tabular}

$\overline{\left.{ }^{a} \text { Reaction conditions: benzophenone (1s, } 5 \mathrm{mmol}\right), \mathbf{2} \text { or } \mathbf{5}(10 \mathrm{mmol}) \text {, super- }}$ fine kaolin $(5 \mu \mathrm{m}, 0.75 \mathrm{mmol})$, PEG-400 $(10 \mathrm{~mL}), 195 \mathrm{~W}$.

在上述条件的基础上，以 4-甲基苯乙酮(1w)、3-硝 基苯乙酩 $(1 x)$ 、苯丙酩 $(1 y)$ 、二苯甲酮 $(1 \mathrm{~s})$ 为原料与乙酰 氧肟酸(5)进行肟交换反应, 得到相应的肟类化合物, 结 果如表 4 所示. 由表 4 可以看出, 微波辐射条件下, 苯丙 酮与乙酰氧肟酸的反应产率较低(表 4, Entry 3). 苯环上 的电子效应对反应没有明显的影响, 无论是吸电子取代 (例如硝基)还是供电子取代基(例如甲基), 得到的产率
都在中等左右.

表 4 肜类化合物 $2 \mathrm{w}, 2 \mathrm{x}, 2 \mathrm{y}$ 和 $2 \mathrm{~s}$ 的合成

Table 4 Synthesis of oxime compounds $2 \mathrm{w}, 2 \mathrm{x}, 2 \mathrm{y}$ and $2 \mathrm{~s}$

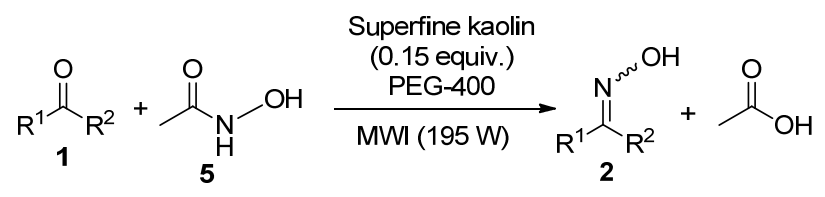

\begin{tabular}{cclcc}
\hline Entry & \multicolumn{1}{c}{$\mathrm{R}^{1}$} & \multicolumn{1}{c}{$\mathrm{R}^{2}$} & Product & Isolated yield ${ }^{a} \%$ \\
\hline 1 & $\mathrm{CH}_{3}$ & $4-\mathrm{CH}_{3}-\mathrm{C}_{6} \mathrm{H}_{4}$ & $\mathbf{2 w}$ & 59 \\
2 & $\mathrm{CH}_{3}$ & $3-\mathrm{NO}_{2}-\mathrm{C}_{6} \mathrm{H}_{4}$ & $\mathbf{2 x}$ & 63 \\
3 & $\mathrm{C}_{2} \mathrm{H}_{5}$ & $\mathrm{C}_{6} \mathrm{H}_{5}$ & $\mathbf{2 y}$ & 42 \\
4 & $\mathrm{C}_{6} \mathrm{H}_{5}$ & $\mathrm{C}_{6} \mathrm{H}_{5}$ & $\mathbf{2 s}$ & 67 \\
\hline
\end{tabular}

${ }^{a}$ Reaction conditions: $1(5 \mathrm{mmol}), 2(10 \mathrm{mmol})$, superfine kaolin $(5 \mu \mathrm{m}, 0.75$ mmol), PEG-400 (10 mL), $195 \mathrm{~W}$.

\section{5 肜醚类化合物的制备}

在以上肜类化合物的合成基础上，以酮 $(5 \mathrm{mmol})$, 甲氧基胺盐酸盐或 $O$-芐基羟胺盐酸盐 $(10 \mathrm{mmol})$ 为原料, 在 PEG-400 $(10 \mathrm{~mL})$ 溶液中再加入超细高岭土 $(5 \mu \mathrm{m}$, $0.75 \mathrm{mmol}$ ), 在 $195 \mathrm{~W}$ 的微波辐射下制备得到 3 种肜醚 类化合物, 收率中等, 结果见 Scheme 1.

\section{6 超细高岭土的回收}

从表 5 可以看出在循环使用 5 次超细高岭土的回收 率保持在 $89 \%$ \% $73 \%$, 但是随着使用次数的增加产物收 率总体呈下降趋势, 其原因可能是催化剂中杂质的增多 和活性成分流失, 造成催化能力的降低.

表 5 超细高岭土的回收

Table 5 Recovery of superfine kaolin

\begin{tabular}{cccc}
\hline $\begin{array}{c}\text { Recovery } \\
\text { No. }\end{array}$ & $\begin{array}{c}\text { Recovery of super- } \\
\text { fine kaolin/g }\end{array}$ & $\begin{array}{c}\text { Addition of super- } \\
\text { fine kaolin } / \mathrm{g}\end{array}$ & $\begin{array}{c}\text { Isolated } \\
\text { yield }^{a} \%\end{array}$ \\
\hline 0 & 0.19 & & 92 \\
1 & 0.17 & 0.02 & 79 \\
2 & 0.16 & 0.03 & 53 \\
3 & 0.14 & 0.05 & 61 \\
4 & 0.16 & 0.03 & 54 \\
\hline
\end{tabular}

${ }^{a}$ Reaction conditions: $1(5 \mathrm{mmol})$, hydroxylamine hydrochloride $(10 \mathrm{mmol})$, superfine kaolin $(5 \mu \mathrm{m}, 0.75 \mathrm{mmol})$, PEG-400 $(10 \mathrm{~mL}), 195 \mathrm{~W}$.

\section{7 缩合反应机理推测}

超细高岭土晶体的单元结构中含有一个四面体配 位氧化硅层和一个八面体配位氧化铝层, 四面体层由四 个与氧等距的硅原子组成, 四面体顶尖指向氧化铝层并 与它共享氧原子; 八面体层的分子式为 $\mathrm{Al}_{2}(\mathrm{OH})_{6}$, 其结 构类似水铝矿物，羟基两个朝上，两个朝下，中间各间 隔两个氧 ${ }^{[34,45 \sim 48]}$. 反应可能的反应机理 ${ }^{[49]}$ 见 Scheme 2.

首先超细高岭土中的氧化硅作为路易斯酸中心, 活 化羰基, 使得羰基中心碳上电子云密度减少, 有利于亲 核试剂羟胺的进攻, 再经过质子迁移, 脱除催化剂超细 
<smiles>CONCCl</smiles><smiles>CONC(Cl)Cl</smiles>

3

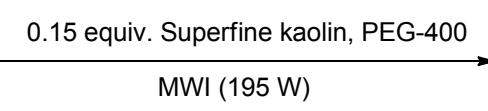

$\underset{\mathrm{MWI}(195 \mathrm{~W})}{\stackrel{0.15 \text { equiv. Superfine kaolin, PEG-400 }}{\longrightarrow}}$<smiles>CO/N=C/c1ccc(Cl)cc1Cl</smiles>

$8 a$

$72 \%$ yield<smiles>CON=C(C)c1cccc(C(C)=NOC)c1</smiles>

$8 \mathrm{~b}$

$59 \%$ yield<smiles>Oc1ccc2/c(=N\OCc3ccccc3)c3ccccc3oc2c1</smiles>

$8 \mathrm{c}$

$68 \%$ yield

图式 1 肟醚类化合物的合成

Scheme 1 Synthesis of oxime ethers
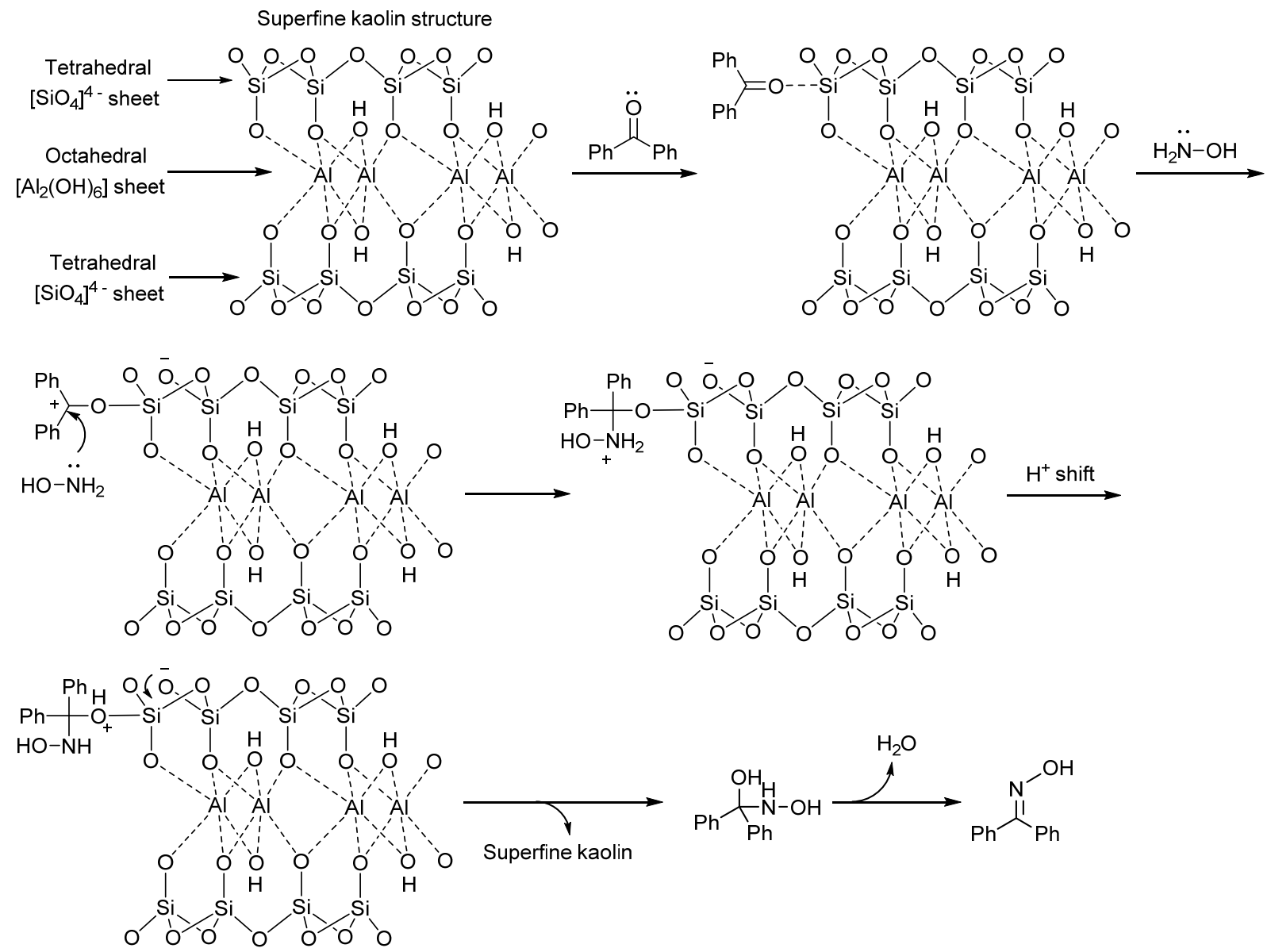

图式 2 可能的反应机理

Scheme 2 Proposed mechanism of the reaction

高岭土, 得到差胺化的二苯甲酮, 再经脱水, 得到二苯 甲酮豚。

\section{2 结论}

在微波辐射的条件下进行了羰基化合物与盐酸着 
胺的反应，得到了一系列肜类化合物，通过对不同反应 条件的探索, 确定了最佳反应条件: 当超细高岭土用量 为 0.15 equiv., 微波输出功率为 $195 \mathrm{~W}$, 反应时间为 5 min, PEG-400 为溶剂时, 产率最高可达 $92 \%$. 与通常肜 类化合物的合成方法相比，该方法大幅度缩短了反应时 间, 提高了产物产率, 且后处理简单, 对环境友好, 为 肜类化合物的合成提供了有效方法. 同时在该反应条件 下, 对肜交换反应进行了初步的研究, 发现超细高岭土 适用于催化肟交换反应，但产率不是很高，反应条件有 待今后进一步优化.

\section{3 实验部分}

\section{1 仪器与试剂}

JM-A20002 型电子天平(诸暨市超泽衡器设备有限 公司); WBFY-201 型微波化学反应器(巩义市科瑞仪器 有限公司); ZF-6 型三用紫外分析仪(金坛市盛蓝仪器制 造有限公司); R205 型旋转蒸发器(台州市信力电子设备 有限公司); M-565 型熔点仪(上海沃珑仪器有限公司); Advance III HD 400 型核磁共振波谱仪(瑞士 Bruker 公 司); 5700 型傅里叶红外光谱仪(日本岛津公司); Quattro micro API 型液质联用仪 (美国 Waters 公司); SYNAPT-G2-Si_with_DESI_Angledleft2 型离子淌度四级杆 串联飞行时间质谱仪(美国 Waters 公司). 实验所用试剂 均为市售分析纯或化学纯, 购于阿拉丁试剂(上海)公司 或国药集团化学试剂有限公司，使用前未作处理.

\section{2 肟类化合物 $2 \mathrm{a} \sim 2 \mathrm{v}$ 的制备}

在 $100 \mathrm{~mL}$ 单口烧瓶中加入化合物 $1(5 \mathrm{mmol})$ 、盐 酸着弪胺 $(10 \mathrm{mmol})$ 和 PEG-400 (10 mL), 再加入超细高岭 土 $(0.75 \mathrm{mmol})$, 将单口烧瓶放入微波化学反应器中, 接 上球型冷凝管, 通冷却水, 设置功率为 $195 \mathrm{~W}$, 通过薄 层色谱(TLC)跟踪反应进程. 反应结束后, 过滤除去催 化剂, 冷却至室温, 将滤液转移至分液漏斗, 加入 50 $\mathrm{mL}$ 水和 $30 \mathrm{~mL}$ 乙酸乙酯, 充分振荡分出有机层后, 水 层每次用乙酸乙酯萃取 $(30 \mathrm{~mL} \times 2)$. 合并有机相, 用饱 和食盐水和清水各清洗 1 次, 分出有机层, 无水硫酸钠 干燥, 旋蒸出溶剂, 粗产物用 $95 \%$ 乙醇重结晶, 得目标 产物 $\mathbf{2 a} \sim 2 \mathrm{v}$.

丙酮肜 (2a): 无色晶体，产率 71\%. m.p. 60.9 $62.3{ }^{\circ} \mathrm{C}$ (lit. $\left.{ }^{[50]} 61 \sim 62{ }^{\circ} \mathrm{C}\right) ;{ }^{1} \mathrm{H}$ NMR $\left(400 \mathrm{MHz}, \mathrm{CDCl}_{3}\right)$ $\delta: 1.90(\mathrm{~d}, J=4.8 \mathrm{~Hz}, 6 \mathrm{H}), 9.21(\mathrm{~s}, 1 \mathrm{H})$; IR (KBr) $v: 3354$, 3205, 2922, 2746, 1678, 1498, 1427, 1371, 1068, 813, 613, $480 \mathrm{~cm}^{-1}$; MS (70 eV) m/z (\%): $74\left(\mathrm{M}^{+}, 100\right)$.

丁酮肜 (2b): 无色液体, 产率 $62 \% .{ }^{1} \mathrm{H}$ NMR $(400$ $\left.\mathrm{MHz}, \mathrm{CDCl}_{3}\right) \delta: 1.09$ (t, $\left.J=7.4 \mathrm{~Hz}, 3 \mathrm{H}\right), 1.89$ (d, $J=7.2$ $\mathrm{Hz}, 3 \mathrm{H}$ ), 2.23 (dd, $J=14.8,7.2 \mathrm{~Hz}, 2 \mathrm{H}$ ), 9.88 (s, 1H); IR
(KBr) v: 3255, 2974, 2922, 1654, 1460, 1371, 1240, 1097, 1074, 979, 931, 796, $611 \mathrm{~cm}^{-1}$; MS (70 eV) $\mathrm{m} / z(\%): 88$ $\left(\mathrm{M}^{+}, 100\right)$.

环戊酮肜(2c)：无色晶体，产率 43\%. m.p. 55.4 $56.3{ }^{\circ} \mathrm{C}$ (lit. ${ }^{[51]} 56 \sim 57{ }^{\circ} \mathrm{C}$ ); ${ }^{1} \mathrm{H}$ NMR $\left(400 \mathrm{MHz}, \mathrm{CDCl}_{3}\right.$ ) $\delta: 1.74 \sim 1.76(\mathrm{~m}, 4 \mathrm{H}), 2.41(\mathrm{~d}, J=36.0 \mathrm{~Hz}, 4 \mathrm{H}), 9.64 \sim$ 9.26 (m, 1H); IR (KBr) v: 3221, 3126, 2964, 1691, 1475, $1425,1290,1211,1153,972,941,792,650 \mathrm{~cm}^{-1}$; MS (70 eV) $m / z(\%): 100\left(\mathrm{M}^{+}, 100\right)$.

环己酮肜(2d): 白色固体, 产率 48\%. m.p. 87.8 $90.3{ }^{\circ} \mathrm{C}$ (lit. $\left.{ }^{[52]} 87 \sim 89{ }^{\circ} \mathrm{C}\right) ;{ }^{1} \mathrm{H}$ NMR $\left(400 \mathrm{MHz}, \mathrm{CDCl}_{3}\right)$ $\delta: 1.58 \sim 1.70(\mathrm{~m}, 6 \mathrm{H}), 2.22(\mathrm{t}, J=6.2 \mathrm{~Hz}, 2 \mathrm{H}), 2.51(\mathrm{t}, J=$ $6.2 \mathrm{~Hz}, 2 \mathrm{H}), 8.86$ (brs, 1H); IR (KBr) $v: 3197,3113,2935$, 2893, 2858, 1664, $898 \mathrm{~cm}^{-1}$; MS (70 eV) $\mathrm{m} / z$ (\%): 114 $\left(\mathrm{M}^{+}, 100\right)$.

2-甲基环已酮肜 (2e)：黄色油状液体，产率 $52 \% .{ }^{1} \mathrm{H}$ NMR (400 MHz, $\left.\mathrm{CDCl}_{3}\right) \delta: 1.11(\mathrm{~d}, J=6.0 \mathrm{~Hz}, 3 \mathrm{H}), 1.32$ (t, $J=9.8 \mathrm{~Hz}, 1 \mathrm{H}), 1.55(\mathrm{~d}, J=44.0 \mathrm{~Hz}, 2 \mathrm{H}), 1.76 \sim 1.95$ $(\mathrm{m}, 4 \mathrm{H}), 2.10 \sim 2.34(\mathrm{~m}, 1 \mathrm{H}), 3.10(\mathrm{~d}, J=11.2 \mathrm{~Hz}, 1 \mathrm{H})$, 9.07 (s, 1H); IR (KBr) v: 3284, 2964, 2929, 2858, 1446, 1375, $754 \mathrm{~cm}^{-1}$; MS (70 eV) m/z (\%): $128\left(\mathrm{M}^{+}, 100\right)$.

环庚酮肜(2f): 黄色油状液体, 产率 $63 \%$. ${ }^{1} \mathrm{H}$ NMR $\left(400 \mathrm{MHz}, \mathrm{CDCl}_{3}\right) \delta: 1.24$ (t, $\left.J=7.0 \mathrm{~Hz}, 3 \mathrm{H}\right), 1.57$ (d, $J=$ $2.4 \mathrm{~Hz}, 3 \mathrm{H}), 2.37(\mathrm{t}, J=5.6 \mathrm{~Hz}, 2 \mathrm{H}), 2.58(\mathrm{t}, J=6.0 \mathrm{~Hz}$, 2H), 3.72 (dd, $J=14.0,6.8 \mathrm{~Hz}, 2 \mathrm{H}), 8.95$ (s, 1H); IR (KBr) $v: 3230,3095,2926,2854,2690 \mathrm{~cm}^{-1}$; MS (70 eV) $\mathrm{m} / \mathrm{z}$ (\%): $128\left(\mathrm{M}^{+}, 100\right)$.

苯甲醛肜 $(2 \mathrm{~g})$ : 无色透明液体，产率 $81 \%$. ${ }^{1} \mathrm{H}$ NMR $\left(400 \mathrm{MHz}, \mathrm{CDCl}_{3}\right) \delta: 7.38(\mathrm{t}, J=3.2 \mathrm{~Hz}, 3 \mathrm{H}), 7.58(\mathrm{dd}$, $J=5.6,1.6 \mathrm{~Hz}, 2 \mathrm{H}), 8.18(\mathrm{~d}, J=2.0 \mathrm{~Hz}, 1 \mathrm{H}), 9.15(\mathrm{~d}, J=$ $50.4 \mathrm{~Hz}, 1 \mathrm{H}$ ); IR (KBr) v: 3309, 3062, 2900, 1496, 1448, $1300,1211,954,869,756,692,646 \mathrm{~cm}^{-1}$; MS $(70 \mathrm{eV}) \mathrm{m} / \mathrm{z}$ (\%): $122\left(\mathrm{M}^{+}, 100\right)$.

2-羟基苯甲醛肜 (2h)：黄色固体，产率 86\%. m.p. $56.1 \sim 58.6{ }^{\circ} \mathrm{C}$ (lit. $\left.{ }^{[53]} 57 \sim 59{ }^{\circ} \mathrm{C}\right) ;{ }^{1} \mathrm{H}$ NMR $(400 \mathrm{MHz}$, $\left.\mathrm{CDCl}_{3}\right) \delta: 1.87(\mathrm{~s}, 1 \mathrm{H}), 4.85(\mathrm{~s}, 1 \mathrm{H}), 6.93(\mathrm{t}, J=7.6 \mathrm{~Hz}$, $1 \mathrm{H}), 6.99(\mathrm{~d}, J=8.4 \mathrm{~Hz}, 1 \mathrm{H}), 7.19(\mathrm{dd}, J=8.0,1.6 \mathrm{~Hz}$, $1 \mathrm{H}), 7.27 \sim 7.32(\mathrm{~m}, 1 \mathrm{H}), 8.23(\mathrm{~s}, 1 \mathrm{H})$; IR (KBr) v: 3377, 3062, 1620, 1575, 1474, 1408, 1290, 1261, 1195, 991, 896, 754, 696, $646 \mathrm{~cm}^{-1}$; MS (70 eV) $\mathrm{m} / z(\%): 138\left(\mathrm{M}^{+}, 100\right)$.

4-羟基苯甲醛肜(2i): 黄色固体，产率 42\%. m.p. 90.5 92.7 ${ }^{\circ} \mathrm{C}$ (lit. ${ }^{[54]} 92.0 \sim 93.6{ }^{\circ} \mathrm{C}$ ); ${ }^{1} \mathrm{H}$ NMR $(400$ MHz, DMSO- $\left.d_{6}\right) \delta: 6.78(\mathrm{~d}, J=8.8 \mathrm{~Hz}, 2 \mathrm{H}), 7.41$ (d, $J=$ $8.8 \mathrm{~Hz}, 2 \mathrm{H}), 8.01$ (s, 1H), 9.78 (s, 1H), 10.86 (s, 1H); IR (KBr) $v: 3365,2991,1606,1516,1483,1431,1365,1273$, 
1205, 1161, 1103, 950, 869, 812, $702 \mathrm{~cm}^{-1}$; MS (70 eV) $m / z(\%): 136\left(\mathrm{M}^{-}, 100\right)$.

2,4-二氯苯甲醛肜 $(\mathbf{2 j})$ : 白色固体, 产率 $87 \%$. m.p. $132.7 \sim 134.6{ }^{\circ} \mathrm{C}$ (lit. $\left.{ }^{[5]} 134 \sim 136{ }^{\circ} \mathrm{C}\right) ;{ }^{1} \mathrm{H}$ NMR (400 MHz, DMSO-d $\left.d_{6}\right) \delta: 7.25(\mathrm{t}, J=5.0 \mathrm{~Hz}, 1 \mathrm{H}), 7.42(\mathrm{~d}, J=$ $2.4 \mathrm{~Hz}, 1 \mathrm{H}), 7.73$ (dd, $J=4.8,1.6 \mathrm{~Hz}, 1 \mathrm{H}), 7.79$ (d, $J=8.4$, $1 \mathrm{H}), 8.49$ (s, 1H); IR (KBr) v: 3286, 2922, 1581, 1550, 1479, 1382, 1313, 1207, 1101, 974, 873, 815, 779, 667 $\mathrm{cm}^{-1}$; MS (70 eV) m/z (\%): $188\left(\mathrm{M}^{-}, 100\right), 190\left(\mathrm{M}^{-}, 75\right)$.

2-乙酰基呋喃肟(2k): 淡红色液体, 产率 $42 \% .{ }^{1} \mathrm{H}$ NMR (400 MHz, $\left.\mathrm{CDCl}_{3}\right) \delta: 2.40(\mathrm{~s}, 1 \mathrm{H}), 7.09$ (dd, $J=5.2$, $3.6 \mathrm{~Hz}, 1 \mathrm{H}), 7.49$ (dd, $J=3.6,0.8 \mathrm{~Hz}, 1 \mathrm{H}), 7.55$ (dd, $J=$ 5.2, $0.8 \mathrm{~Hz}, 1 \mathrm{H}), 10.67$ (s, 1H); IR (KBr) v: 3269, 1724, $1635,1577,1483,1427,1284,1165,1118,1074,1010$, $947,885,744,625,594 \mathrm{~cm}^{-1}$; MS (70 eV) $\mathrm{m} / z(\%): 126$ $\left(\mathrm{M}^{+}, 100\right)$.

苯乙酮肟(2l): 白色固体，产率 83\%. m.p. 57.7 $59.9{ }^{\circ} \mathrm{C}$ (lit. ${ }^{[56]} 56 \sim 58{ }^{\circ} \mathrm{C}$ ); ${ }^{1} \mathrm{H}$ NMR $\left(400 \mathrm{MHz}, \mathrm{CDCl}_{3}\right.$ ) $\delta: 2.31(\mathrm{~s}, 3 \mathrm{H}), 7.38(\mathrm{dd}, J=4.4,1.2 \mathrm{~Hz}, 3 \mathrm{H}), 7.62(\mathrm{dd}, J=$ 7.6, 4.0 Hz, 2H), 9.60 (s, 1H); IR (KBr) v: 3292, 3248, 2922, 1643, 1446, $1369 \mathrm{~cm}^{-1}$; MS (70 eV) m/z (\%): 136 $\left(\mathrm{M}^{+}, 100\right)$.

4-差基苯乙酮肜 $(\mathbf{2 m})$ : 白色针晶, 产率 $87 \%$. m.p. 143.2 146.4 ${ }^{\circ} \mathrm{C}$ (lit. $\left.{ }^{[57]} 146 \sim 147{ }^{\circ} \mathrm{C}\right) ;{ }^{1} \mathrm{H}$ NMR (400 MHz, DMSO- $\left.d_{6}\right) \delta: 2.09$ (s, 3H), 6.77 (d, $\left.J=2.4 \mathrm{~Hz}, 2 \mathrm{H}\right)$, 7.48 (t, $J=4.2 \mathrm{~Hz}, 2 \mathrm{H}), 9.60(\mathrm{~s}, 1 \mathrm{H}), 10.84$ (s, 1H); IR (KBr) v: 3329, 3265, 3067, 2791, 2566, 1639, 1606, 1516, 1442, 1382, 1365, $1238 \mathrm{~cm}^{-1}$; MS (70 eV) m/z (\%): 152 $\left(\mathrm{M}^{+}, 100\right)$.

4-甲氧基苯乙酮肜(2n): 白色固体, 产率 84\%. m.p. 83.9 86.7 ${ }^{\circ} \mathrm{C}$ (lit. ${ }^{[58]} 82 \sim 85{ }^{\circ} \mathrm{C}$ ); ${ }^{1} \mathrm{H}$ NMR (400 MHz, $\left.\mathrm{CDCl}_{3}\right) \delta: 2.28(\mathrm{~s}, 3 \mathrm{H}), 3.82(\mathrm{~s}, 3 \mathrm{H}), 6.90(\mathrm{~d}, J=8.8 \mathrm{~Hz}$, 2H), 7.57 (d, $J=9.2 \mathrm{~Hz}, 2 \mathrm{H}), 9.42(\mathrm{~s}, 1 \mathrm{H})$; IR (KBr) $v$ : 3296, 3244, 3076, 2960, 2922, 1608, 1452, 1369, 1303, 1249, $1020 \mathrm{~cm}^{-1}$; MS (70 eV) m/z (\%): $166\left(\mathrm{M}^{+}, 100\right)$.

4-硝基苯乙酮肜(2o): 淡黄色固体, 产率 87\%. m.p. $172.7 \sim 176.0{ }^{\circ} \mathrm{C}$ (lit. $\left.{ }^{[59]} 169 \sim 171{ }^{\circ} \mathrm{C}\right) ;{ }^{1} \mathrm{H}$ NMR $(400$ MHz, DMSO- $\left.d_{6}\right) \delta: 2.22(\mathrm{~s}, 3 \mathrm{H}), 7.92(\mathrm{~d}, J=8.8 \mathrm{~Hz}, 2 \mathrm{H})$, 8.24 (d, $J=8.8 \mathrm{~Hz}, 2 \mathrm{H}), 11.78$ (s, 1H); IR (KBr) v: 3250, $3109,3069,1597,1512,1338,852 \mathrm{~cm}^{-1}$; MS (70 eV) $\mathrm{m} / \mathrm{z}$ (\%): $181\left(\mathrm{M}^{+}, 98\right)$.

4-氯苯乙酮肟 $(2 p)$ : 白色固体, 产率 $83 \%$. m.p. $101.1 \sim 102.8 \quad{ }^{\circ} \mathrm{C} \quad\left(\right.$ lit. $\left.{ }^{[60]} 97 \sim 99 \quad{ }^{\circ} \mathrm{C}\right) ;{ }^{1} \mathrm{H}$ NMR $(400$ $\left.\mathrm{MHz}, \mathrm{CDCl}_{3}\right) \delta: 2.28(\mathrm{~s}, 3 \mathrm{H}), 7.35(\mathrm{~d}, J=8.8 \mathrm{~Hz}, 2 \mathrm{H})$, 7.56 (d, $J=8.8 \mathrm{~Hz}, 2 \mathrm{H}), 9.18$ (s, 1H); IR (KBr) v: 3294,
3234, 3062, 2924, 1641, 1593, 1460, 1369, $1097 \mathrm{~cm}^{-1}$; MS (70 eV) m/z (\%): $170\left(\mathrm{M}^{+}, 100\right), 172\left(\mathrm{M}^{+}, 37\right), 192(68)$, 197 (22).

3-乙酰基吡啶肟(2q): 淡黄色固体, 产率 77\%. m.p. $116.3 \sim 117.2{ }^{\circ} \mathrm{C}$ (lit. $\left.{ }^{[52]} 116 \sim 118{ }^{\circ} \mathrm{C}\right) ;{ }^{1} \mathrm{H}$ NMR $(400$ MHz, DMSO- $\left.d_{6}\right) \delta: 2.19(\mathrm{~d}, J=2.8 \mathrm{~Hz}, 3 \mathrm{H}), 2.23(\mathrm{~s}, 1 \mathrm{H})$, $7.43(\mathrm{dd}, J=8.0,4.8 \mathrm{~Hz}, 1 \mathrm{H}), 8.00 \sim 8.05(\mathrm{~m}, 1 \mathrm{H}), 8.57$ (dd, $J=4.8,1.6 \mathrm{~Hz}, 1 \mathrm{H}), 8.85(\mathrm{~d}, J=1.2 \mathrm{~Hz}, 1 \mathrm{H})$; IR (KBr) v: 3423, 3174, 3064, 2964, 2877, 1726, 1579, 1467, 1375, 1282, 1124, 1072, 1006, 981, 929, 806, 744, 705 $\mathrm{cm}^{-1}$; MS (70 eV) m/z (\%): $137\left(\mathrm{M}^{+}, 100\right)$.

$(E, E)$-3-丁基-2-酮基-4-苯基肜 $[(E, E)-2 \mathbf{r}]$ : 白色固体, 产率 48\%. m.p. $121.3 \sim 123.5{ }^{\circ} \mathrm{C}$ (lit. ${ }^{[61]} 116{ }^{\circ} \mathrm{C}$ ); ${ }^{1} \mathrm{H}$ NMR (400 MHz, $\left.\mathrm{CDCl}_{3}\right) \delta: 2.16(\mathrm{~d}, J=1.2 \mathrm{~Hz}, 3 \mathrm{H}), 6.88$ (d, $J=4.0 \mathrm{~Hz}, 2 \mathrm{H}), 7.29$ (d, $J=7.2 \mathrm{~Hz}, 1 \mathrm{H}), 7.35$ (t, $J=7.4$ $\mathrm{Hz}, 3 \mathrm{H}), 7.47$ (d, $J=7.2 \mathrm{~Hz}, 2 \mathrm{H})$; IR (KBr) v: 3261, 3207, 3032, 2889, 2358, 1625, 1492, 1446, 1371, 1328, 1305, 1205, 1074, 1020, $960 \mathrm{~cm}^{-1}$; MS (70 eV) m/z (\%): 146 $(\mathrm{M}-15,100), 162\left(\mathrm{M}^{+}, 49\right)$.

$(Z, E)$-3-丁基-2-酮基-4-苯基肜 $[(Z, E)-2 \mathbf{r}]$ : 白色固体, 产率 40\%. m.p. $107.4 \sim 109.5{ }^{\circ} \mathrm{C}$ (lit. $\left.{ }^{[62]} 109{ }^{\circ} \mathrm{C}\right) ;{ }^{1} \mathrm{H}$ NMR (400 MHz, $\left.\mathrm{CDCl}_{3}\right) \delta: 2.15(\mathrm{~d}, J=1.6 \mathrm{~Hz}, 3 \mathrm{H}) ; 6.88$ (dd, $J=6.8,4.0 \mathrm{~Hz}, 2 \mathrm{H}), 7.29$ (d, $J=7.2 \mathrm{~Hz}, 1 \mathrm{H}), 7.35$ (t, $J=7.2 \mathrm{~Hz}, 3 \mathrm{H}), 7.47$ (d, $J=7.6 \mathrm{~Hz}, 2 \mathrm{H})$; IR (KBr) $v$ : $3265,3209,3032,2922,2360,1625,1577,1492,1446$, 1371, 1328, 1307, 1261, 1205, 1159, 1074, 1026, 960 $\mathrm{cm}^{-1}$; MS (70 eV) m/z (\%): $146(\mathrm{M}-15,44), 162\left(\mathrm{M}^{+}\right.$, 100).

二苯甲酮肜 (2s): 白色固体，产率 $92 \%$. m.p. $140.7 \sim 142.3{ }^{\circ} \mathrm{C}$ (lit. $\left.{ }^{[63]} 143 \sim 144{ }^{\circ} \mathrm{C}\right) ;{ }^{1} \mathrm{H}$ NMR $(400$ $\left.\mathrm{MHz}, \mathrm{CDCl}_{3}\right) \delta: 7.31(\mathrm{dd}, J=6.4,4.8 \mathrm{~Hz}, 2 \mathrm{H}), 7.34 \sim 7.39$ $(\mathrm{m}, 2 \mathrm{H}), 7.41 \sim 7.44(\mathrm{~m}, 2 \mathrm{H}), 7.45(\mathrm{~s}, 2 \mathrm{H}), 7.47(\mathrm{~d}, J=1.2$ $\mathrm{Hz}, 2 \mathrm{H}), 9.00 \sim 9.11(\mathrm{~m}, 1 \mathrm{H})$; IR (KBr) v: 3248, 3055, 2887, 1490, 1446, 1327, 1303, 1159, 1076, 1031, 997, 916 $\mathrm{cm}^{-1}$; MS (70 eV) m/z (\%): $198\left(\mathrm{M}^{+}, 35\right), 220$ (100).

2-氯二苯甲酮肜 $(\mathbf{2 t})$ : 白色固体，产率 $89 \%$. m.p. $125.7 \sim 127.2{ }^{\circ} \mathrm{C} ;{ }^{1} \mathrm{H}$ NMR $\left(400 \mathrm{MHz}, \mathrm{CDCl}_{3}\right) \delta: 7.26 \sim$ $7.28(\mathrm{~m}, 1 \mathrm{H}), 7.31(\mathrm{dd}, J=6.4,4.8 \mathrm{~Hz}, 1 \mathrm{H}), 7.34 \sim 7.37$ $(\mathrm{m}, 2 \mathrm{H}), 7.38 \sim 7.41(\mathrm{~m}, 2 \mathrm{H}), 7.45 \sim 7.47(\mathrm{~m}, 2 \mathrm{H}), 7.51 \sim$ $7.54(\mathrm{~m}, 1 \mathrm{H}), 8.66 \sim 8.81(\mathrm{~m}, 1 \mathrm{H})$; IR (KBr) v: 3278, 3244, 3057, 2908, 2360, 1591, 1492, 1446, 1325, 1305, 1255, $1168,1128,1062,1033,1001,931 \mathrm{~cm}^{-1}$; MS (70 eV) $\mathrm{m} / z$ (\%): $216\left(\mathrm{M}-15^{+}, 33\right), 232\left(\mathrm{M}^{+}, 41\right), 254\left(\mathrm{M}+\mathrm{Na}^{+}\right.$, $100)$.

4,4'-二甲氧基二苯甲酮肜 $(\mathbf{2} \mathbf{u})$ : 白色针晶, 产率 
86\%. m.p. $131.1 \sim 133.2{ }^{\circ} \mathrm{C}$ (lit. ${ }^{[64]} 129 \sim 130{ }^{\circ} \mathrm{C}$ ); ${ }^{1} \mathrm{H}$ NMR (400 MHz, $\left.\mathrm{CDCl}_{3}\right) \delta: 3.81(\mathrm{~s}, 3 \mathrm{H}), 3.85$ (s, 3H), 6.84 (d, $J=8.8 \mathrm{~Hz}, 2 \mathrm{H}), 6.97$ (d, $J=8.8 \mathrm{~Hz}, 2 \mathrm{H}), 7.40$ (dd, $J=$ 8.4, $1.2 \mathrm{~Hz}, 4 \mathrm{H}$ ), 8.99 (brs, 1H); IR (KBr) v: 3265, 3053, 3003, 2943, 2904, 2835, 1604, 1512, 1444, 1301, 1251, 1174, 1121, 1029, 993, $923 \mathrm{~cm}^{-1}$; MS (70 eV) $\mathrm{m} / z(\%)$ : $243\left(\mathrm{M}^{+}, 89\right), 265\left(\mathrm{M}+\mathrm{Na}^{+}, 99\right)$.

4,4'-二氟二苯甲酮肜 $(\mathbf{2 v})$ : 橘黄色固体, 产率 $91 \%$. m.p. $133.8 \sim 135.7{ }^{\circ} \mathrm{C}$ (lit. ${ }^{[15]}$ : $\left.133 \sim 135{ }^{\circ} \mathrm{C}\right) ;{ }^{1} \mathrm{H}$ NMR $\left(400 \mathrm{MHz}, \mathrm{CDCl}_{3}\right) \delta: 7.00 \sim 7.05(\mathrm{~m}, 2 \mathrm{H}), 7.13 \sim 7.18(\mathrm{~m}$, $2 \mathrm{H}), 7.40 \sim 7.44(\mathrm{~m}, 4 \mathrm{H}), 8.82(\mathrm{~s}, 1 \mathrm{H})$; IR (KBr) v: 3338, 3049, 2912, 1598, 1508, 1226, 1155, 1099, 991, $921 \mathrm{~cm}^{-1}$; MS (70 eV) $m / z$ (\%): 219 (97).

\section{3 醛、酮活性比较}

在 $100 \mathrm{~mL}$ 单口烧瓶中投入苯甲醛 $(5 \mathrm{mmol})$ 、二苯 甲酮 $(5 \mathrm{mmol})$ 、盐酸着弪胺 $(10 \mathrm{mmol})$ 和 PEG-400 $(10 \mathrm{~mL})$, 再加入超细高岭土 $(5 \mu \mathrm{m}, 0.75 \mathrm{mmol})$, 将单口烧瓶放入 微波化学反应器中, 后续实验操作过程参照 3.2 节缩合 反应制备化合物 $\mathbf{2} \mathbf{a} \sim \mathbf{2 v}$ 的实验过程, 得苯甲酫肜 $(\mathbf{2 g})$, 无色透明液体, 产率 78\%.

在 $100 \mathrm{~mL}$ 单口烧瓶中投入 1,3 -二乙酰基苯(4 $\mathrm{mmol}$ )、盐酸着弪胺 $(12 \mathrm{mmol})$ 和 PEG-400 $(10 \mathrm{~mL})$, 再加入 超细高岭土 $(0.75 \mathrm{mmol})$, 将单口烧瓶放入微波化学反应 器中, 后续实验操作过程参照 3.2 节缩合反应制备化合 物 $\mathbf{2 a} \sim 2 \mathbf{w}$ 的实验过程, 得 1,1'-(1,3-亚苯基)二乙氧基二 肜(4a): 白色固体, 产率 91\%. m.p. 203.3 205.7 ${ }^{\circ} \mathrm{C}$ (lit. ${ }^{[65]} 206 \sim 208{ }^{\circ} \mathrm{C}$ ); ${ }^{1} \mathrm{H}$ NMR (400 MHz, DMSO- $d_{6}$ ) $\delta$ : 2.18 (s, 6H), 7.41 (t, $J=7.8 \mathrm{~Hz}, 1 \mathrm{H}), 7.64$ (dd, $J=8.0,1.6$ $\mathrm{Hz}, 2 \mathrm{H}), 7.94$ (s, 1H), 11.26 (s, 2H); IR (KBr) v: 3213, 3140, 3097, 2962, 2920, 1465, 1367, 1325, 1217, 1103, $1104,948,916,823,794,746,684 \mathrm{~cm}^{-1}$; MS (70 eV) $\mathrm{m} / \mathrm{z}$ (\%): $191\left(\mathrm{M}^{-}, 90\right)$.

\section{4 肟交换反应底物 2 或 5 的篮选}

在 $100 \mathrm{~mL}$ 单口烧瓶中投入二苯甲酮 $(5 \mathrm{mmol})$, 化 合物 2 或 $5(10 \mathrm{mmol})$ 和 PEG-400 $(10 \mathrm{~mL})$, 再加入超细 高岭土 $(5 \mu \mathrm{m}, 0.75 \mathrm{mmol})$, 将单口烧瓶放入微波化学反 应器中, 后续实验操作过程参照 3.2 节缩合反应制备化 合物 $2 \mathrm{a} \sim 2 \mathrm{v}$ 的实验过程, 粗产物柱层析分离(洗脱剂比 例为 $V$ (乙酸乙酯) $: V$ (石油醚) $=1: 19)$, 得目标产物 $\mathbf{2 s}$.

\section{5 肟交换反应制备化合物 $2 \mathrm{~s}$ 和 $2 \mathrm{w} \sim 2 \mathrm{y}$}

在 $100 \mathrm{~mL}$ 单口烧瓶中投入酮 $(5 \mathrm{mmol})$, 乙酰氧肜 酸 $(10 \mathrm{mmol})$ 和 PEG-400 (10 mL), 再加入超细高岭土(5 $\mu \mathrm{m}, 0.75 \mathrm{mmol}$ ), 将单口烧瓶放入微波化学反应器中, 后续实验操作过程参照 3.2 节缩合反应制备化合物 2a
$2 \mathbf{v}$ 的实验过程，粗产物柱层析分离[洗脱剂：乙酸乙酯/ 石油醚, $V: V=1: 19$ (2s、2w), $1: 13$ (2x), $1: 7$ (2y)], 得目标产物 $2 \mathrm{~s}$ 和 $\mathbf{2 w} \sim 2 \mathrm{y}$.

二苯甲酮肜 $(2 \mathrm{~s})$ : 白色固体，产率 67\%.

4-甲基苯乙酮肪 $(2 \mathrm{w})$ : 白色固体，产率 59\%. m.p. 89.3 91.6 ${ }^{\circ} \mathrm{C}$ (lit. $\left.{ }^{[60]} 85 \sim 87{ }^{\circ} \mathrm{C}\right) ;{ }^{1} \mathrm{H}$ NMR $(400 \mathrm{MHz}$, $\left.\mathrm{CDCl}_{3}\right) \delta: 2.28$ (s, 3H), $2.36(\mathrm{~s}, 3 \mathrm{H}), 7.18(\mathrm{~d}, J=8.0 \mathrm{~Hz}$, 2H), $7.51(\mathrm{~d}, J=8.0 \mathrm{~Hz}, 2 \mathrm{H}), 9.71(\mathrm{~s}, 1 \mathrm{H})$; IR (KBr) $v$ : 3213, 3126, 3059, 2914, 1510, 1435, $1365 \mathrm{~cm}^{-1}$; MS (70 eV) $m / z(\%): 150\left(\mathrm{M}^{+}, 100\right)$.

3-硝基苯乙酮肜(2x): 白色固体, 产率 63\%. m.p. 130.2 132.5 ${ }^{\circ} \mathrm{C}$ (lit. $\left.{ }^{[66]} 131 \sim 132{ }^{\circ} \mathrm{C}\right) ;{ }^{1} \mathrm{H}$ NMR (400 $\left.\mathrm{MHz}, \mathrm{CDCl}_{3}\right) \delta: 2.34$ (s, 3H), $7.57(\mathrm{t}, J=8.2 \mathrm{~Hz}, 1 \mathrm{H}), 7.99$ (d, $J=8.0 \mathrm{~Hz}, 1 \mathrm{H}), 8.23$ (dd, $J=8.0,1.6 \mathrm{~Hz}, 2 \mathrm{H}), 8.50$ (s, 1H); IR (KBr) v: 3232, 3145, 3082, 2918, 1525, 1352, 800, $788 \mathrm{~cm}^{-1}$; MS (70 eV) m/z (\%): $181\left(\mathrm{M}^{+}, 99\right)$.

1-苯基丙酮肪 $(2 \mathrm{y})$ : 黄色油状液体, 产率 $42 \% .{ }^{1} \mathrm{H}$ NMR (400 MHz, $\left.\mathrm{CDCl}_{3}\right) \delta: 1.74(\mathrm{t}, J=5.2 \mathrm{~Hz}, 3 \mathrm{H}), 2.83$ (dd, $J=10.4, \mathrm{~Hz}, 2 \mathrm{H}), 7.34 \sim 7.44(\mathrm{~m}, 3 \mathrm{H}), 7.60 \sim 7.61(\mathrm{~m}$, 2H), 9.94 (brs, 1H); IR (KBr) v: 3302, 3201, 3143, 2821, 2360, 2058, 1701, 1608, 1537, 1382, 1321, 1271, 1222, $1128,1049,823,717 \mathrm{~cm}^{-1}$; MS (70 eV) $\mathrm{m} / z(\%): 150\left(\mathrm{M}^{+}\right.$, 97).

\section{6 肜醚类化合物 $8 a \sim 8 c$ 的制备}

在 $100 \mathrm{~mL}$ 单口烧瓶中投入酮类化合物 $(5 \mathrm{mmol})$, 甲氧基胺盐酸盐或 $O$-芐基羟胺盐酸盐 $(10 \mathrm{mmol})$ (当投入 的酮类化合物为 1,3-二乙酰基苯时, 所用的甲氧基胺盐 酸盐为 $15 \mathrm{mmol})$ 和 PEG-400 $(10 \mathrm{~mL})$, 再加入超细高岭 土 $(5 \mu \mathrm{m}, 0.75 \mathrm{mmol})$, 将单口烧瓶放入微波化学反应器 中, 后续实验操作过程参照 3.2 节缩合反应制备化合物 2a $\sim 2 \mathrm{v}$ 的实验过程, 粗产物柱层析分离[洗脱剂比例为 $V($ 乙酸乙酯) $： V($ 石油醚 $)=0 ： 1(\mathbf{8 a}), 1: 25(\mathbf{8 b}), 1: 2$ $(\mathbf{8 c}))$, 得目标产物 $8 \mathrm{a} \sim 8 \mathrm{c}$.

2,4-二氯苯甲醛- $O$-甲基肪 $(\mathbf{8 a})$ : 白色固体, 产率 $72 \%$. m.p. 62.1 64.2 ${ }^{\circ} \mathrm{C}$ (lit. ${ }^{[67]} 63 \sim 65{ }^{\circ} \mathrm{C}$ ); ${ }^{1} \mathrm{H}$ NMR $\left(400 \mathrm{MHz}, \mathrm{CDCl}_{3}\right) \delta: 3.99$ (s, 3H), 7.24 (dd, $J=8.4,1.6$ $\mathrm{Hz}, 1 \mathrm{H}), 7.39$ (d, $J=2.0 \mathrm{~Hz}, 1 \mathrm{H}), 7.83(\mathrm{~d}, J=4.4 \mathrm{~Hz}, 1 \mathrm{H})$, 8.41 (s, 1H); IR (KBr) v: 3086, 2970, 2929, 2814, 1589, 1548, 1467, 1379, 1336, 1205, 1145, 1043, 925, 862, 819 $\mathrm{cm}^{-1}$; MS (70 eV) m/z (\%): $204\left(\mathrm{M}^{+}, 100\right), 206\left(\mathrm{M}^{+}+2\right.$, 76).

1-(3-(1-甲氧亚氨基-乙基)苯基)乙酮- $O$-甲基肜 $(\mathbf{8 b})$ : 淡黄色油状液体, 产率 59\%. ${ }^{1} \mathrm{H}$ NMR $\left(400 \mathrm{MHz}, \mathrm{CDCl}_{3}\right)$ $\delta$ : $2.24(\mathrm{~s}, 6 \mathrm{H}), 4.00(\mathrm{~s}, 6 \mathrm{H}), 7.35(\mathrm{t}, J=7.8 \mathrm{~Hz}, 1 \mathrm{H}), 7.63$ (dd, $J=7.6,1.6 \mathrm{~Hz}, 2 \mathrm{H}), 7.90(\mathrm{~d}, J=1.2 \mathrm{~Hz}, 1 \mathrm{H}) ;{ }^{13} \mathrm{C}$ 
NMR $\left(100 \mathrm{MHz}, \mathrm{CDCl}_{3}\right) \delta: 13.14,76.46,124.04,126.92$, $128.00,128.41,128.57,136.94,138.18,154.83$; IR (KBr) v: 3066, 2937, 2899, 2818, 1612, 1575, 1463, 1436, 1369, 1334, 1294, 1273, 1055, 914, 889, 833, 796, 748, 694, 648 $\mathrm{cm}^{-1}$; HRMS calcd for $\mathrm{C}_{12} \mathrm{H}_{16} \mathrm{O}_{2} \mathrm{~N}_{2}$ 221.1290, found 221.1293 .

3-羟基- $9 H$-呫吨-9-酮- $O$-芐基肜 $(\mathbf{8 c})$ : 黄色油状液 体, 产率 68\%. ${ }^{1} \mathrm{H}$ NMR $\left(400 \mathrm{MHz}, \mathrm{CDCl}_{3}\right) \delta: 0.97 \sim 1.02$ $(\mathrm{m}, 2 \mathrm{H}), 4.08 \sim 4.13(\mathrm{~m}, 1 \mathrm{H}), 5.21 \sim 5.24(\mathrm{~m}, 2 \mathrm{H}), 7.31 \sim$ $7.38(\mathrm{~m}, 5 \mathrm{H}) 7.41 \sim 7.44(\mathrm{~m}, 2 \mathrm{H}), 7.58(\mathrm{~s}, 2 \mathrm{H}), 8.13 \sim 8.16$ $(\mathrm{m}, 1 \mathrm{H}) ;{ }^{13} \mathrm{C} \mathrm{NMR}(100 \mathrm{MHz}, \mathrm{CDCl} 3) \delta: 76.49,127.15$, $127.18,128.07,128.52,128.54,128.76,128.94,129.93$, $131.04,132.26,132.29,132.42,137.54,137.59,149.12$, 167.79; IR (KBr) v: 3136, 2970, 2929, 2814, 1589, 1467, $1379,1205,1045,925,862,817,561 \mathrm{~cm}^{-1}$; HRMS calcd for $\mathrm{C}_{19} \mathrm{H}_{13} \mathrm{O}_{3} \mathrm{~N}$ 317.3399, found 301.1423.

\section{7 超细高岭土的回收}

反应结束后使其自然冷却至室温, 过滤出催化剂, 并用少量乙酸乙酯洗涤滤饼. 将所得固体加入 $20 \mathrm{~mL}$ 的 乙酸乙酯中搅拌 $3 \mathrm{~h}$, 减压抽滤, 再用少量乙酸乙酯和 水清洗滤饼, 将固体置于烘箱中, $100{ }^{\circ} \mathrm{C}$ 下烘干 $2 \mathrm{~h}$, 再 置于马弗炉中, $500{ }^{\circ} \mathrm{C}$ 下焙烧 $2 \mathrm{~h}$, 回收超细高岭土.

\section{辅助材料(Supporting Information) 产物 $2 \mathrm{a} \sim 2 \mathrm{v}, \mathbf{4 a}$ 和} $\mathbf{8 a} \sim 8 \mathbf{c}$ 的 ${ }^{1} \mathrm{H}$ NMR, IR 和 MS 谱图. 这些材料可以免费 从本刊网站(http://sioc-journal.cn/)上下载.

\section{References}

[1] Bolotin, D. S.; Bokach, N. A.; Demakova, M. Y.; Kukushkin, V. Y. Chem. Rev. 2017, 117, 13039.

[2] Ran, L. F.; Liang, H.; Guan, Z. H. Chin. J. Org. Chem. 2013, 33, 66 (in Chinese). (再陇飞, 梁浩, 关正辉, 有机化学, 2013, 33, 66.)

[3] Watson, K. G. Aust.J. Chem. 2011, 64, 367.

[4] Kolesnikov, A. M.; Yuidin, M. A.; Nikiforov, A. S.; Ivanov, I. M.; Vengerovich, N. G.; Makacheev, A. S. Bull. Exp. Biol. Med. 2018, 164,624 .

[5] Wang, X. B.; Chen, M. H.; Li, Q.; Zhang, J. P.; Ruan, X. H.; Xie, Y.; Xue, W. Chem. Pap. 2017, 71, 1225.

[6] Canario, C.; Silvestre, S.; Falcao, A.; Alves, G. Curr. Med. Chem. 2018, 25, 660

[7] Patil, V. V.; Gayakwad, E. M.; Shankarling, G. S. J. Org. Chem. 2016, $81,781$.

[8] Liu, S. H.; Hao, F.; Liu, P. L.; Luo, H. A. RSC Adv. 2015, 5, 22863.

[9] Reddy, M. K.; Mallik, S.; Ramakrishna, I.; Baidya, M. Org. Lett. 2017, 19, 1694.

[10] Hong, Z.; Li, J. J.; Chen, G.; Jiang, H. J.; Yang, X. F.; Pan, H.; Su, W. K. RSC Adv. 2016, 6, 13581.

[11] Sathyanarayana, P.; Upare, A.; Ravi, O.; Muktapuram, P. R.; Bathula, S. R. RSC $A d v$. 2016, 6, 22749.

[12] Bezlada, A.; Szewczyk, M.; Mlynarski, J. J. Org. Chem. 2016, 81, 336.

[13] Xu, Y. Y.; Yang, Q. S.; Li, Z. H.; Gao, L. Y.; Zhang, D. S.; Wang, S. F.; Zhao, X. Q.; Wang, Y. J. Chem. Eng. Sci. 2016, 152, 717.
[14] Lin, C. K.; Cheng, L. W.; Li, H. Y.; Yun, W. Y.; Cheng, W. C. Org. Biomol. Chem. 2015, 13, 2100.

[15] Xie, F. K.; Du, C.; Pang, Y. D.; Lian, X.; Xue, C. T.; Chen, Y. Y.; Wang, X. F.; Cheng, M. S.; Guo, C.; Lin, B.; Liu, Y. X. Tetrahedron Lett. 2016, 57, 5820 .

[16] Peterson, K. E.; Cinelli, M. A.; Morrell, A. E.; Mehta, A.; Dexheimer, T. S.; Agama, K.; Antony, S.; Pommier, Y.; Cushman, M. J. Med. Chem. 2011, 54, 4937.

[17] Eshghi, H.; Hassankhani, A. Org. Prep. Proced. Int. 2005, 37, 575.

[18] Sharghi, H.; Sarvari, M. H. Synlett 2001, 1, 99.

[19] Yao, Q. C.; Fan, L.; Liu, L.; Huang, Y. M.; Cui, J. G. Chem. Res. Appl. 2013, 25, 220 (in Chinese).

(姚秋翠, 范否, 刘亮, 黄燕敏, 崔建国, 化学研究与应用, 2013, 25,220 .)

[20] Noverges, B.; Mollar, C.; Medio-Simón, M.; Asensio, G. Adv. Synth. Catal. 2013, 355, 2327.

[21] Manetti, F.; Magnani, M.; Castagnolo, D.; Passalacqua, L.; Botta, M.; Corelli, F.; Saddi, M.; Deidda, D.; De Logu, A. ChemMedChem 2006, 1,973 .

[22] Ghozlojeh, N. P.; Setamdideh, D. Orient. J. Chem. 2015, 31, 1823.

[23] Juskowiak, M.; Krzyżanowski, P. J. Prakt. Chem. 1989, 331, 870.

[24] Shimaoka, H.; Kuramoto, H.; Furukawa, J. I.; Miura, Y.; Kurogochi, M.; Kita, Y.; Hinou, H.; Shinohara, Y.; Nishimura, S. I. Chem.-Eur. J. 2007, 13, 1664.

[25] Sridhar, M.; Narsaiah, C.; Raveendra, J.; Reddy, G. K.; Reddy, M. K. K.; Ramanaiah, B. C. Tetrahedron Lett. 2011, 52, 4701.

[26] Hyodo, K.; Togashi, K.; Oishi, N.; Hasegawa, G.; Uchida, K. Green Chem. 2016, 18, 5788 .

[27] Pienkoß, F.; Ochoa-Hernández, C.; Theyssen, N.; Leitner, W. ACS Sustainable Chem. Eng. 2018, 6, 8782.

[28] Aras, A.; Albayrak, M.; Arikan, M.; Sobolev, K. Clay Miner. 2007, $42,233$.

[29] Zheng, Y. F.; Li, D.; Chen, S. K.; Sun, S. H.; Liu, C. H.; Gao, X. H. Ind. Catal. 2012, 20, 1 (in Chinese). (郑云锋, 李荻, 陈淑琨, 孙书红, 刘从华, 高雄厚, 工业催化, 2012, 20, 1.)

[30] Shen, J. X.; Ma, H. W. Bull. Chin. Ceram. Soc. 2016, 35, 1150 (in Chinese).

(申继学，马鸿文，硅酸盐通报，2016，35, 1150.)

[31] Yan, H. Q.; Chen, X. Q.; Wu, T. T.; Feng, Y. H.; Wang, C. X.; Li, J. C.; Lin, Q. Polym. Bull. 2014, 71, 2923.

[32] Ignatova, T.; Mincheva, K.; Ignatov, S.; Dzhelyaydinova, A.; Petkov, T.; Kyazimov, A. J. Chem. Technol. Metall. 2013, 48, 186.

[33] Liu, H. J.; Ao, L. Inner Mongolia Pet. Ind. 1998, 24, 112 (in Chinese).

(刘惠军, 敖丽, 内蒙古石油化工, 1998, 24, 112.)

[34] Chermahini, A. N.; Teimouri, A.; Momenbeik, F.; Zarei, A.; Dalirnasab, Z.; Ghaedi, A.; Roosta, M. J. Heterocycl. Chem. 2010, 47, 913.

[35] Maheen, G.; Tian, G.; Song, Z.; He, C.; Shi, Z.; Liu, Z.; Yuan, H.; Feng, S. J. Heterocycl. Chem. 2010, 47, 483.

[36] Hirano, M.; Monobe, H.; Yakabe, S.; Morimoto T. J. Chem. Res., Synop. 1998, 0, 662

[37] Matsuzaki, T.; Ohsuga, K.; Sugi, Y.; Takami, Y.; Imamura, J. J. Chem. Soc. Jpn., Chem. Ind. Chem. 1985, 12, 2331.

[38] Roudier, J. F.; Foucaud, A. Tetrahedron Lett. 1984, 25, 4375.

[39] Kappe, C. O.; Pieber, B.; Dallinger, D. Angew. Chem., Int. Ed. 2013, $52,1088$.

[40] Nuechter, M.; Ondruschka, B.; Bonrath, W.; Gum, A. Green Chem. 2004, 6, 128 .

[41] Alam, M.; Lee, D. U. Korean J. Chem. Eng. 2015, 32, 1142

[42] Erdogan, T.; Erdogan, F. O. Lett. Org. Chem. 2018, 15, 99.

[43] Khan, A. U.; Avecillia, F.; Malik, N.; Khan, M. S.; Khan, M. S.; Mushtaque, M. J. Mol. Struct. 2016, 1122, 100.

[44] Gedye, R. N.; Smith, F. E.; Westaway, K. C. Can. J. Chem., 1988, $66,17$.

[45] Lu, C. B. China Pulp Pap. Ind. 2017, 38, 11 (in Chinese). (路崇斌，中华纸业, 2017, 38, 11.) 
[46] Lapides, I.; Yariv, S.; Lahav, N. Clay Miner. 1995, 30, 287.

[47] Gu, T.; Zou, Z. G. B. Bull. Chin. Ceram. Soc. 2005, 4, 70 (in Chinese).

(顾涛，邹正光，硅酸盐通报， 2005, 4, 70.)

[48] Tian, J. Crystal Chemistry of Silicate, Wuhan University Press, Wuhan, 2010, pp. 194 196 (in Chinese). (田键, 硅酸盐晶体化学, 武汉大学出版社, 武汉, 2010, pp. 194 196.)

[49] Xiong, W.; Liu, J.; Huang, N.; Zhang, H. P. J. Shaoyang Univ. (Nat. Sci. Ed.) 2012, 9, 75 (in Chinese).

(熊文, 刘静, 黄娜, 张红萍, 邵阳学院学报(自然科学版), 2012, 9,75.)

[50] Lieberman, S. V. J. Am. Chem. Soc. 1955, 77, 1114.

[51] Zhukovskaya, N. A.; Dikusar, E. A. Russ. J. Org. Chem. 2010, 46, 180.

[52] Ngwerume, S.; Camp, J. E. J. Org. Chem. 2010, 75, 6271.

[53] Fazaeli, R.; Tangestaninejad, S.; Aliyan H. Catal. Commun. 2007, $8,205$.

[54] Chen, J. B.; Liu, E. M.; Chern, T. R.; Yang, C. W.; Lin, C. I.; Huang, N. K.; Lin, Y. L.; Chern, Y.; Lin, J. H.; Fang, J. M. ChemMedChem 2011, 6, 1390

[55] Wiley, R. H.; Wakefield, B. J. J. Org. Chem. 1960, 25, 546
[56] Mokhtari, J.; Naimi-Jamal, M. R.; Hamzeali, H.; Dekamin, M. G.; Kaupp, G. ChemSusChem 2009, 2, 248.

[57] Corma, A.; Garcia, H.; Leyva, A. J. Mol. Catal. A-Chem. 2005 , 230,97

[58] Wang, H. Y.; Mueller, D. S.; Sachwani, R. M.; Londino, H. N.; Anderson, L. L. Org. Lett. 2010, 12, 2290.

[59] Balogh, A.; Kovendi, A.; Rotaru, D.; Craciunescu, E. US 3117995 , 1964 [Chem Abstr. 1964, 60, 52549].

[60] Prateeptongkum, S.; Jovel, I.; Jackstell, R.; Vogl, N.; Weckbecker, C.; Beller, M. Chem. Commun. 2009, 1990.

[61] Talapatra, S. K.; Chaudhuri, P.; Talapatra, B. Heterocycles 1980, 14 1279.

[62] Domnin, N. A.; Yakimovich, S. I. Zh. Neorg. Khim. 1965, 1, 1024.

[63] Jain, N.; Kumar, A.; Chauhan, S. M. S. Tetrahedron Lett. 2005, 46, 2599.

[64] Gunatilaka, A. A. L.; Ramachandran, S. Indian J. Chem. 1978, 16B, 432.

[65] Bruton, E. A.; Brammer, L.; Pigge, F. C.; Aakeroey, C. B.; Leinen, D. S. New J. Chem. 2003, 27, 1084

[66] Bodor, N.; Fey, L.; Kovendi, A. Rev. Roum. Chim. 1966, 11, 405.

[67] Dubost, E. ; Fossey, C.; Cailly, T. Rault, S.; Fabis, F. J. Org. Chem. 2011, 76, 6414. 